\title{
5 The Emergence of Heterotrophy in an Eco-evolutionary Model \\ Modelling Trophic Transitions in a Resource-based Framework with Naturally-bounded Trait Distributions
}

\author{
Roger Cropp · John Norbury
}

1 Received: date / Accepted: date

\begin{abstract}
A plankton eco-evolutionary model with an alga that has the metabolic pathways to allow it to function as an autotroph or heterotroph is considered. Ecological constraints dictate that the traits that describe the feeding preferences and abilities of the alga naturally have bounded distributions. The trait distributions are then non-normal, and evolve with the population as it changes its trophic behaviour from an autotroph to a heterotroph.

A key result of the simulations is that the populations remain in ecological stasis for many generations while the trait mean slowly adapts - only at the conclusion of this transition does herbivory emerge. After initially adapting to improve its competitive performance as an autotroph, the adapting population eventually emerges as a heterotroph having maximised its share of the resources at the expense of its prey, previously its competitor.
\end{abstract}

Keywords eco-evolution · resource-based model · gamma-distributed traits · naturally-bounded traits

\section{Introduction}

Predation is thought to have evolved many times, at different trophic levels, and is acknowledged as a strong evolutionary force that can shape ecosystems (Bengtson 2002). While it was originally assumed that evolutionary change in populations progressed at a much slower pace than changes due to interactions with their biotic and abiotic environment, the recent view is that evolution can progress rapidly, on similar time scales to ecological dynamics (Schoener 2011). Substantial evidence exists to support this view (Pelletier et al. 2009), with experimental

R. Cropp

School of Environment and Science, Griffith University, Nathan, Queensland, 4111, Australia Tel.: +61 737354036

E-mail: r.cropp@griffith.edu.au

J. Norbury

Mathematical Institute, University of Oxford, Andrew Wiles Building, ROQ, Woodstock Road, Oxford OX2 6GG, UK 
evolution even being demonstrated in laboratory studies (Post \& Palkovacs 2009). Competition and predation systems separately have strong theoretical support, with histories dating back nearly 100 years, and the coevolution of predators and prey has received considerable attention (Abrams 1991, Cortez \& Ellner 2010, Cortez 2016, Pastor 2017). However, few models exist to demonstrate the processes by which populations transition from one trophic strategy to another, in particular how autotrophs might transition to heterotrophy (Govaert et al. 2018, De Meester et al. 2019).

Marine plankton are perhaps canonical examples of where understanding the processes by which such transitions occur may be of immediate practical importance (Fussman et al. 2002). Mixotrophy, the trophic interaction where plants behave like animals and animals behave like plants and the distinction between a plant and an animal becomes blurred, is now known to be common amongst the marine plankton, with approximately $80 \%$ of species thought to be functional mixotrophs (Flynn et al. 2013). The key role of marine plankton in the transfer of gas between the ocean and the atmosphere, including the production of oxygen, the drawdown of carbon dioxide, and the production of climatically important gases such as dimethyl sulphide (Mitra et al. 2014), suggests that an improved theoretical understanding of how populations transition between these roles is central to developing climate models that can incorporate these processes.

The evolution of a new trophic strategy proceeds via the modification of an existing strategy - the evolution of heterotrophy in an organism depends upon the modification of a metabolic system, such as the Krebs cycle, previously used by an autotroph (Bell 2012). Experimental results have demonstrated the transition of an alga from autotrophy to heterotrophy in the laboratory over ecological time, evidenced by their ability to survive in the dark solely by metabolising the organic molecule acetate (Bell 2012). This transition involves an intermediate stage known as mixotrophy, a common strategy amongst many organisms (Selosse et al. 2017), but well documented in marine plankton in particular (Flynn et al. 2013). Bell (2012) notes that the green alga he studied, Chlamydomonas, is a unicellular chlorophyte that can use both autotrophic and heterotrophic metabolic pathways. Despite these being located in different cellular compartments, changes in the strategy used involve dramatic adjustments in the economy of the cell. Chlamydomonas grows rapidly in light by fixing $\mathrm{CO}_{2}$ and more slowly in dark by metabolizing acetate. The initial populations in the experiments could not survive solely by metabolizing acetate, but around $8 \%$ evolved to become pure heterotrophs within a few tens of generations and lost the ability to grow in light. Bell (2012) attributed the majority of the change in trait variation in Chlamydomonas in the experiments to selection.

Eco-evolutionary modelling that uses a quantitative genetics approach considers how the average value of a population trait, that has some variance within the population, changes in response to changes in the population's biotic and abiotic environment (Abrams 1991, Cortez \& Ellner 2010, Fussman et al. 2007, Pastor 2017). These "quantitative genetics" approaches consider evolution resulting from existing genetic variation (Govaert et al. 2018). Such approaches are often based on a description of how traits evolve derived by Lande (1976), using a variety of functions that estimate how changes in the average value of the trait affects the population fitness (Abrams et al. 1993, Fussman et al. 2002, Cortez 2016, Pastor 2017). These fitness functions are often simple growth functions derived from 
heuristic Lotka-Volterra models of the population interactions. We continue this approach here, but with the key difference that we consider eco-evolutionary processes in a resource-based Lotka-Volterra model that naturally includes bounds upon the distributions of key traits.

The bounds imply that the trait variance is related to the trait mean, and consequently that trait distributions may be more accurately modelled by bounded distributions such as $\beta$ (Klauschies et al. 2018) or $\gamma$ (Cropp \& Norbury 2019) rather than normal distributions (Abrams 1999, Abrams \& Matsuda 2004, Chesson 2000, Cortez \& Ellner 2010, Cortez \& Patel 2017). Unlike normal distributions in which the mean is independent of the variance, these are related in bounded distributions, a property observed in some traits of real populations (Gaedke \& Klauschies 2017). This leads to a new eco-evolutionary model that possesses significantly different time scale behaviours. We use such a model to show that an autotrophic alga can evolve to a heterotroph. Interestingly, much of the adaptation occurs during periods of ecological stasis, when the alga develops its ability to function as a heterotroph, but continues to actually function almost exclusively as an autotroph. Eventually, it relatively rapidly switches its trophic strategy from autotrophy to heterotrophy by losing its autotrophic ability.

\section{The Eco-evolutionary Model}

We consider a simple Lotka-Volterra ecosystem model of two interacting populations in a resource-based framework that has been described in detail elsewhere (Cropp \& Norbury 2015b), so here we mostly describe the evolutionary aspects of the model. However, a key component of the approach we take is that the resource-based framework in which we construct the ecological sub-model provides the natural bounds on the trait distributions in the evolutionary sub-model, so we provide a very brief summary of the "ecological axioms" that underpin the model:

- The resources and populations are measured in a common currency, usually the limiting nutrient recycling in the system;

- The limiting nutrient is available in a finite total amount, which is used to scale the populations so that each resource or population is measured as a fraction of the total limiting nutrient;

- The mass of limiting nutrient is conserved as it flows through the ecosystem;

- The per-capita rate of change of each population is independent of how we measure the system, and depends only on the available resource and the other population fractions;

- The growth of every population is an explicit, monotonic function of the resources available to it, whether inorganic nutrient or another population - when a population's resources are maximal it increases, and when they are zero it decreases;

- When the inorganic nutrient pool is empty, no resource can be taken from the pool, and the limiting resource in the pool can only increase.

These conditions ensure that the ecosystem model equations have sensible properties, but it is the fifth condition that is particularly important in an eco-evolutionary context. This condition prevents the model populations from being immortal (if 
they can increase when they have no resources); and relieves us from modelling populations that are never viable (if they can't increase when resources are maximal). These simple conditions provide the bounds on the trait distributions that turn out to be vital to the eco-evolutionary processes in this model. This is discussed in detail in $\S 2.1$.

We consider an autotroph population $x_{1}$ and a mixotroph population $x_{2}$ in the Conservative Normal framework (Cropp \& Norbury 2015b). The populations are measured in terms of the fraction of the total limiting nutrient in the ecosystem that they contain. The mixotroph population $x_{2}$ initially functions as an autotroph, but has a "feeding preference" parameter $0 \leq \rho \leq 1$. This parameter identifies the relative preference for $x_{2}$ of autotrophy (via photosynthesis) or heterotrophy (by predating upon $x_{1}$ ) and thus captures whether it "does better" by autotrophy or heterotrophy. The evolutionary component of the model assumes that $x_{1}$ does not adapt to the change in $x_{2}$ 's feeding behaviour, and allows the trait average food preference $\rho$ of $x_{2}$ to vary as well as $a_{21}$, the trait average of its direct interaction with $x_{1}$. Competition between $x_{1}$ and $x_{2}$ for inorganic nutrient is represented by $a_{21}>0$, while predation of $x_{2}$ upon $x_{1}$ is represented by $a_{21}<0$.

The eco-evolutionary model, obtained by coupling the resource-based LotkaVolterra ecosystem equations with the Lande evolution equations (see below), is described by the equations:

$$
\begin{aligned}
\frac{d x_{1}}{d t} & =x_{1} f_{1}\left(x_{1}, x_{2}, N\right)=x_{1}\left(r_{1} N-m_{1}-a_{11} x_{1}-a_{12} x_{2}\right), \\
\frac{d x_{2}}{d t} & =x_{2} f_{2}\left(x_{1}, x_{2}, N, \rho\right)=x_{2}\left(r_{2} \rho N-m_{2}-a_{21}(1-\rho) x_{1}-a_{22} x_{2}\right), \\
\frac{d a_{21}}{d t} & =-h_{1}^{2}\left(\frac{a_{21}+a_{12}}{\beta_{1}}\right)(1-\rho) x_{1} \\
\frac{d \rho}{d t} & =h_{2}^{2}\left(\frac{(1-\rho) \rho}{\beta_{2}^{2}}\right)\left(r_{2} N+a_{21} x_{1}\right) .
\end{aligned}
$$

Here, $r_{1}$ and $r_{2}$ are the average autotrophic growth rates of $x_{1}$ and $x_{2}$ respectively on inorganic nutrient $N, m_{1}$ and $m_{2}$ are their average density-independent mortality rates, $a_{11}$ and $a_{22}$ are their average density-dependent mortality rates, and $a_{12}$ and $a_{21}$ are the average effects of each population upon the other. The fitness function $\ln \bar{W}=f_{2}$ (see equation (4) below) has been used to derive the evolutionary equations, and we have assumed that $a_{21}$ is bounded only by the local mass balance constraint $-a_{12}<a_{21}$ (loosely that $x_{2}$ cannot gain more mass through predation than $x_{1}$ loses), and that $0 \leq \rho \leq 1$ by definition. The system is considered at steady state with its environment, so that net movements of mass of limiting nutrient across the boundary are zero (Vitousek \& Matson 2012). The system is scaled by the total mass of recycled limiting nutrient, so the relation $N=1-x_{1}-x_{2}$ holds. This defines both an ecospace $E \equiv\left\{0<x_{1}<1,0<x_{2}<1,0<x_{1}+x_{2}<1\right\}$ in which the ecologically valid dynamics of the system occur, and a resource space $R \equiv\left\{0<N<1,0<x_{1}<1,0<N+x_{1}<1\right\}$ that contains all the resources for the two populations. Note that the system is not closed with respect to flows of energy and other nutrients.

We follow the derivation of Lande (1976) and consider the evolution of the average value of a heritable phenotypic character $\bar{z}$, a trait, in response to selection. 
When the phenotype has a $\gamma$-distribution, rather than the commonly assumed normal distribution, the average trait value is given by $\bar{z}(t)=\alpha / \beta$ where $\alpha$ is a shape parameter and $\beta$ is a rate parameter that together determine the distribution. The general form of the $\gamma$-distribution assumes a lower bound of zero, but parameters in resource-limited models may have lower bounds other than zero (equations (6) and $(7))$.

We derive an equation describing how the average value of a trait that is $\gamma$ distributed changes over time in (Cropp \& Norbury 2019). Allowing for a non-zero lower bound $z_{L}$ for $\bar{z}$ and, assuming continuous trait variation, we get:

$$
\frac{d \bar{z}}{d t}=h^{2}\left(\frac{\bar{z}(t)-z_{L}}{\beta}\right) \frac{\partial \ln \bar{W}}{\partial \bar{z}(t)},
$$

where $h^{2}$ is the realised additive heritability of the character and $\bar{W}$ is the average fitness of the population. The consequence of having a bounded trait is that the variance becomes a function of the average trait value (specifically $\sigma^{2}=\left(\bar{z}-z_{L}\right) / \beta$ ) and ensures that phenotypes with bounded distributions are not able to exceed their bounds during computer simulations. Together, the heritability of the trait $\left(h^{2}\right)$, the variance of the trait values $\left(\left(\bar{z}-z_{L}\right) / \beta\right)$, and the magnitude of the sensitivity of the average population fitness to the average trait value $(\partial \ln \bar{W} / \partial \bar{z})$, determine how quickly the average trait value changes. The trait mean and variance are always positive, so the sign of the sensitivity determines whether the trait value increases or decreases.

Where average trait values are bounded both above and below, the distribution of the trait values may be bimodal (Coutinho et al. 2016, Cortez \& Patel 2017), perhaps comprised of reflected $\gamma$-distributions, and then we use the equation:

$$
\frac{d \bar{z}}{d t}=h^{2}\left(\frac{\left(z_{U}-\bar{z}(t)\right)\left(\bar{z}(t)-z_{L}\right)}{\beta^{2}}\right) \frac{\partial \ln \bar{W}}{\partial \bar{z}(t)} .
$$

Note that a $\beta$-distribution might alternately be assumed here (Klauschies et al. 2018).

When considering heuristic models (in which much detail has been abstracted and subsumed into generic descriptions of processes) it is appropriate to use heuristic representations of average population fitness (Abrams et al. 1993, Fussman et al. 2002, Cortez 2016, Pastor 2017). Many of these approaches define the average population fitness to be part or all of the population growth function $f_{i}$. Simply defining the fitness function $\bar{W}_{i}=f_{i}$ is not appropriate for an eco-evolutionary model as the logarithm of the population fitnesses would be undefined at the ecological coexistence steady state (where $f_{i}=0$ for all $i$ ). Here we use the exponential form (Vasseur et al. 2011, Osmond et al. 2017):

$$
\ln \bar{W}_{i}=f_{i} \Rightarrow \bar{W}_{i}=e^{f_{i}} .
$$

As this metric of fitness is an exponential function, it never equals zero within the ecospace and only has optima at the bounds of its range, so the ecological equilibrium (defined by $f_{1}=0=f_{2}$ ) and the fitness equilibrium (defined by $\left.d a_{21} / d t=0=d \rho / d t\right)$ cannot coincide while the trait variance is non-zero. This produces the property that the evolutionary component of the model will continually exert pressure to move the ecological equilibrium to a higher fitness evolutionary state as long as there remains some variance in the population. 


\subsection{Ecological Constraints on Trait Values}

We consider ecologically valid definitions of viable populations that are not immortal (Cropp \& Norbury 2015b): if a population does not have any resource available it reduces; if the world is full of its resource the population increases. We are interested in modelling populations in the heuristic Lotka-Volterra framework, where populations have monotonically increasing per capita growth rates as their resources increase. This ecological axiom may be expressed very succinctly mathematically as:

$$
\left.f_{i}\right|_{R_{i}=1}>0>\left.f_{i}\right|_{R_{i}=0},
$$

where $R_{i}$ is the resource(s) that population $x_{i}$ depends upon for survival. Note that $R_{i}$ may be a living or nonliving resource, for example, in the model (1), the resource for population $x_{1}$ is $R_{1}=N$, as it is purely an autotroph, whereas the resource for population $x_{2}$ is $R_{2}=N+x_{1}$, as it may function as an autotroph (consumes $N$ only), a mixotroph (consumes both) or a heterotroph (consumes $x_{1}$ only).

Populations that do not comply with these constraints are either immortal if $\left.f_{i}\right|_{R_{i}=0} \geq 0$, or unviable if $\left.f_{i}\right|_{R_{i}=1} \leq 0$. When populations function as autotrophs $(\rho=1)$ they depend on $N$ to be able to grow. The maximum resource for both populations $N=1$ is available at the origin of the ecospace $\{0,0\}$ and the minimum resource for both populations occurs on the boundary of the ecospace where $x_{1}+$ $x_{2}=1$. Applying this axiom to (1) and evaluating $f_{1}$ and $f_{2}$ at the respective resource extremes $N=0\left(x_{1}+x_{2}=1\right)$ and $N=1\left(x_{1}=0=x_{2}\right)$, the vertices of the triangular resource space $R$ bounded by $x_{1}=0, x_{2}=0$ and $x_{1}+x_{2}=1 \Rightarrow N=0$ provides naturally arising constraints on the values that the $x_{1}$, and initially $x_{2}$ when it functions as an autotroph, parameters can take:

$$
0<r_{1}-m_{1} \text { with }-m_{1}<a_{11}, a_{12} \text {, and } 0<r_{2}-m_{2} \text { with }-m_{2}<a_{21}, a_{22} \text {. }
$$

Note that in general, $a_{11}>0$ and $a_{22}>0$ as normally increases in the $x_{1}$ and $x_{2}$ populations reduce the availability of resources for further growth, and hence have a detrimental effect upon their own population. Hence, most populations have a detrimental effect upon the growth rate of their own population, commonly referred to as density-dependent or quadratic mortality.

However, when $x_{2}$ functions as a heterotroph $(\rho=0)$, it solely depends upon $x_{1}$ to be able to grow. Evaluating $f_{2}$ at the respective resource extremes $x_{1}=1$ and $x_{1}=0$ (i.e. $\left.f_{2}\right|_{x_{1}=1, \rho=0}>0$ and $\left.f_{2}\right|_{x_{1}=0, \rho=0}<0$ ), leads to the parameter constraints:

$$
-a_{12}<a_{21}<-m_{2}<0<a_{22} .
$$

Here we have included the local mass balance constraint $\left(-a_{21}<a_{12}\right)$ that ensures that $x_{2}$ cannot gain more mass from $x_{1}$ than $x_{1}$ loses. Note that autotrophic growth is zero when $\rho=0$ (consequently the value of $r_{2}$ is not considered), and $a_{22} \geq 0$ is taken as few populations have negative density-dependent mortality. When $x_{2}$ functions as a heterotroph, its maximum growth rate on $x_{1}, a_{21}$, must exceed its density-independent mortality rate $m_{2}$, as stipulated by equation (7), for it to be a viable population. 
The attributes that identify $x_{2}$ as a mixotroph are:

$$
0<m_{2}<r_{2} \text { with } 0<m_{2}<-a_{21}<a_{12},
$$

where $r_{2}>m_{2}$ indicates that $x_{2}$ is able to grow via autotrophy and $m_{2}<-a_{21}<$ $a_{12}$ identifies that it can grow via heterotrophy (Cropp \& Norbury 2015a). As $x_{2}$ develops its mixotrophic ability $a_{21}$ reduces and becomes negative. When it utilises its heterotrophic ability $\rho$ reduces, and when $\rho=0, x_{2}$ has ceased autotrophy and has adapted to become a pure heterotroph.

\subsection{Eco-evolutionary Coupling}

The litmus test that a model is truly eco-evolutionary, in which the dynamics of the ecosystem sub-model influence the evolution sub-model, and the dynamics of the evolution sub-model influence the ecosystem sub-model (Schoener 2011), is that all the blocks of the Jacobian, or community, matrix of the model evaluated at the eco-evolutionary equilibrium point $\left\{x_{1}^{*}, x_{2}^{*}, a_{21}^{*}, \rho^{*}\right\}$ are populated. Here, $0<x_{1}^{*}, x_{2}^{*}<1,0<x_{1}^{*}+x_{2}^{*}<1,-a_{12} \leq a_{21}^{*}$, and $0 \leq \rho^{*} \leq 1$. The locations and stabilities of the equilibrium points are discussed in $\S 2.3$ below and defined explicitly in Appendix 7.1.

The Jacobian matrix of the eco-evolutionary model (1) is:

$J=\left[\begin{array}{cccc}-\left(r_{1}+a_{11}\right) x_{1}^{*} & -\left(r_{1}+a_{12}\right) x_{1}^{*} & 0 & 0 \\ -\left[r_{2} \rho^{*}+a_{21}^{*}\left(1-\rho^{*}\right)\right] x_{2}^{*} & -\left(r_{2} \rho^{*}+a_{22}\right) x_{2}^{*} & \left(1-\rho^{*}\right) x_{1}^{*} x_{2}^{*} & \left(r_{2} N^{*}+a_{21}^{*} x_{1}^{*}\right) x_{2}^{*} \\ -h_{1}^{2}\left(\frac{a_{21}^{*}+a_{12}}{\beta_{1}}\right)\left(1-\rho^{*}\right) & 0 & -h_{1}^{2}\left(\frac{1}{\beta_{1}}\right)\left(1-\rho^{*}\right) x_{1}^{*} & h_{1}^{2}\left(\frac{a_{21}^{*}+a_{12}}{\beta_{1}}\right) x_{1}^{*} \\ h_{2}^{2}\left(\frac{\left(1-\rho^{*}\right) \rho^{*}}{\beta_{2}^{2}}\right)\left(a_{21}^{*}-r_{2}\right)-h_{2}^{2}\left(\frac{\left(1-\rho^{*}\right) \rho^{*}}{\beta_{2}^{2}}\right) r_{2} & h_{2}^{2}\left(\frac{\left(1-\rho^{*}\right) \rho^{*}}{\beta_{2}^{2}}\right) x_{1}^{*} & h_{2}^{2}\left(\frac{1-2 \rho^{*}}{\beta_{2}^{2}}\right)\left(r_{2} N^{*}+a_{21}^{*} x_{1}^{*}\right)\end{array}\right]$,

where, $N^{*}=1-x_{1}^{*}-x_{2}^{*}$.

The upper right $2 \times 2$ block of the Jacobian matrix, that captures how the dynamics of the evolutionary system influences the ecological dynamics, reveals that the evolutionary dynamics do not directly affect the dynamics of the $x_{1}$ population, as expected, but that the dynamics of the $x_{2}$ population are directly affected. The lower left $2 \times 2$ block of the Jacobian matrix, that captures how the ecological dynamics influences the evolutionary dynamics, reveals that the dynamics of the ecological system directly influence the dynamics of the evolutionary system, so the model (1) is fully coupled in an eco-evolutionary context.

\subsection{Equilibrium Points}

The location and stability of the equilibrium points are the key to understanding the dynamics of the eco-evolutionary system (1). The ecosystem component of the model (1) has several ecological equilibrium points that are unstable due to the parameter constraints (6) and (7). The ecosystem component of the model has a coexistence equilibrium point $\left\{x_{1}^{E}, x_{2}^{E}\right\}$, that is relevant to the dynamics of the system, that is not dependent on whether the evolutionary processes are at equilibrium (see below): 


$$
\begin{aligned}
& \left.x_{1}^{E}\right|_{\left\{a_{21}, \rho\right\}}=\frac{r_{1}\left(m_{2}+a_{22}\right)-r_{2} \rho\left(m_{1}+a_{12}\right)-m_{1} a_{22}+m_{2} a_{12}}{a_{11} a_{22}-a_{12} a_{21}(1-\rho)-r_{1}\left(a_{21}(1-\rho)-a_{22}\right)+r_{2} \rho\left(a_{11}-a_{12}\right)}, \\
& \left.x_{2}^{E}\right|_{\left\{a_{21}, \rho\right\}}=\frac{r_{1}\left(1-x_{1}^{E}\right)-m_{1}-a_{11} x_{1}^{E}}{r_{1}+a_{12}} .
\end{aligned}
$$

This point exists in the ecospace $E$ of ecologically valid solutions and is locally stable in the ecological space for the parameter values used in the simulations (Table 1). As the time scales of the ecological dynamics are generally shorter than those of the evolutionary processes, we might expect the populations to generally be close to their equilibrium values, so $x_{1}^{*}$ and $x_{2}^{*}$ are very near to $x_{1}^{E}$ and $x_{2}^{E}$ respectively as $a_{21}$ and $\rho$ vary.

The full eco-evolutionary system has two equilibrium points related to the ecological coexistence equilibrium point given by equation (10). The full details of the locations and eigenvalues of these points are provided in Appendix 7.1; in brief their locations are:

$$
\begin{aligned}
& E P_{0} \equiv\left\{x_{1}^{*}=\left.x_{1}^{E}\right|_{\left\{-a_{12}, 0\right\}}, x_{2}^{*}=\left.x_{2}^{E}\right|_{\left\{-a_{12}, 0\right\}}, a_{21}^{*}=-a_{12}, \rho^{*}=0\right\}, \\
& E P_{1} \equiv\left\{x_{1}^{*}=\left.x_{1}^{E}\right|_{\left\{a_{21}^{*}, 1\right\}}, x_{2}^{*}=\left.x_{2}^{E}\right|_{\left\{a_{21}^{*}, 1\right\}},-a_{21}^{*}<a_{12}, \rho^{*}=1\right\} .
\end{aligned}
$$

Note that if $\rho^{*}=1, x_{1}^{E}$ and $x_{2}^{E}$ do not depend on $a_{21}$, and $\frac{d a_{21}}{d t}=0=\frac{d \rho}{d t}$ for any $a_{21}$. All valid $-a_{21}^{*}<a_{12}$ with $\rho^{*}=1$ form a (half) line of equilibrium points in the eco-evo space, upon which $E P_{1}$ lies. When $\rho^{*}=0, a_{21}$ does affect the ecosystem equilibrium and $\frac{d a_{21}}{d t}=0$ forces $a_{21}^{*}=-a_{12}$, producing the only isolated eco-evolutionary equilibrium point.

Each of $E P_{0}$ and $E P_{1}$ have two eigenvalues $\lambda_{1,2}$ associated with the ecological dynamics (see Appendix 7.1) that are always negative for the parameter values used in the simulations. The third and fourth eigenvalues of these points, that are associated with the evolutionary dynamics, are:

$$
\begin{aligned}
& E P_{0}: \quad \lambda_{3}=-\frac{h_{1}^{2}}{\beta_{1}} x_{1}^{*}, \\
& \lambda_{4}=\frac{h_{2}^{2}}{\beta_{2}^{2}}\left(r_{2} N^{*}-a_{12} x_{1}^{*}\right), \\
& E P_{1}: \quad \lambda_{3}=0, \\
& \lambda_{4}=-\frac{h_{2}^{2}}{\beta_{2}^{2}}\left(r_{2} N^{*}+a_{21}^{*} x_{1}^{*}\right) .
\end{aligned}
$$

$E P_{1}$ has a zero eigenvalue associated with the $a_{21}$ manifold, so the stability of the singular line $E P_{1}$ is always degenerate in the $a_{21}$ direction, but is always stable with respect to $x_{1}, x_{2}$ variation.

The fourth eigenvalue of $E P_{1}$ is negative, and $E P_{1}$ is stable in this direction, for $x_{1}^{*}>\frac{r_{2}\left(1-x_{2}^{*}\right)}{r_{2}+a_{12}}$, and unstable otherwise. Note that the fourth eigenvalues of $E P_{0}$ and $E P_{1}$ are the same magnitude but opposite signs, so when $x_{1}^{*}$ reduces and $E P_{1}$ becomes unstable, $E P_{0}$ simultaneously becomes stable in this direction.

As $\lambda_{3}<0$ always for $E P_{0}$, this point will be globally stable in the ecoevolutionary space when $x_{1}^{*}<\frac{r_{2}\left(1-x_{2}^{*}\right)}{r_{2}+a_{12}}$. Evaluation of the $\lambda_{4}$ eigenvalues with the parameter set used in the numerical simulations (see below) reveals that $E P_{1}$ 
has an attracting $\lambda_{4}$ eigenvalue for the initial part of the simulation, but that $E P_{0}$ is a globally attracting eco-evolutionary equilibrium point for most of the simulations. This suggests that $x_{2}$ initially adapting to become a more effective competitor and reducing the population of $x_{1}$ will change the stability of $E P_{0}$ and initiate its adaptation to heterotrophy.

\subsection{Numerical Simulations}

The model (1) was integrated for 1,000 generations using the parameter values in Table 1 (see Appendix 7.2) starting from the initial conditions $\left\{x_{1}(0)=\right.$ $\left.0.388, x_{2}(0)=0.004, a_{21}(0)=2.00, \rho(0)=0.90\right\}$. Here, $x_{1}$ and $x_{2}$ are $90 \%$ of their equilibrium values $x_{1}^{E}$ and $x_{2}^{E}$. These parameter values and initial conditions comply with the constraints of equation (6) and define the initial state of the system to essentially be competition between two autotrophs. The system is initially dominated by $x_{1}$, and the arrangement of the zero isoclines in Figure 1(a) indicates that without adaptation $x_{1}$ will always dominate the system. However, $0<\rho<1$ reveals that $x_{2}$ has the potential to engage in mixotrophic behaviour. Note we use the term "generation" rather loosely here as the time scale of the model is based on the normalised growth rate of the $x_{1}$ population $\left(r_{1}=1\right.$, which is fixed), but it is the grow rate of the $x_{2}$ population, which varies as $a_{21}$ and $\rho$ vary, that provides the "generations" of interest. The numerical values of the generations should not be interpreted literally, but as indicative of the relative timing of events.

\section{Simulation Results and Discussion}

The adaptation of $x_{2}$ from its initial functioning solely as an autotroph to its final functioning solely as a heterotroph is shown in Figure 1. The initial isocline arrangement and vector field (Figure 1(a)) reveals that the initial parameter set (Table 1) has the two populations coexisting in a stable equilibrium as competing autotrophs. The vector field reveals $x_{1}$ as a relatively strong competitor and $x_{2}$ as a relatively weak competitor, with $x_{1}$ sequestering approximately $40 \%$ of the total nutrient in the system and $x_{2}$ only $0.4 \%$.

The initial response of $x_{2}$ to its rather unfortunate initial condition is to increase both its dependence on autotrophy, revealed by its feeding preference $\rho$ tending to 1 , (Figure 2(e)) and its ability to consume $x_{1}$, revealed by $a_{21}$ becoming negative (Figure 2(b)). Increasing its preference for autotrophy improves $x_{2}$ 's ability to compete with $x_{1}$, and $x_{2}$ rapidly increases its share of the resources from 0.4 to $12 \%$, while $x_{1}$ 's share reduces from 40 to $15 \%$. However, $x_{2}$ remains a poor autotroph competitor, with its intrinsic autotrophic growth $r_{2}$ and mortality properties $m_{2}$ and $a_{22}$ determining that its environmental carrying capacity as an autotroph $K_{2}=\left(r_{2}-m_{2}\right) /\left(r_{2}+a_{22}\right)=0.19$ remains far inferior to that of $x_{1}$ $\left(K_{1}=\left(r_{1}-m_{1}\right) /\left(r_{1}+a_{11}\right)=0.49\right)$.

Figure $2(\mathrm{~b})$ shows that $a_{21}$ rapidly changes from positive to negative values, indicating that the $x_{2}$ population adapts to utilise its metabolic pathways to consume $x_{1}$ relatively quickly. This produces a change in nomenclature for $x_{2}$ 's potential trophic behaviour, from an autotroph to a potential green mixotroph (that grows mostly by autotrophy) to a potential red mixotroph (that grows mostly 

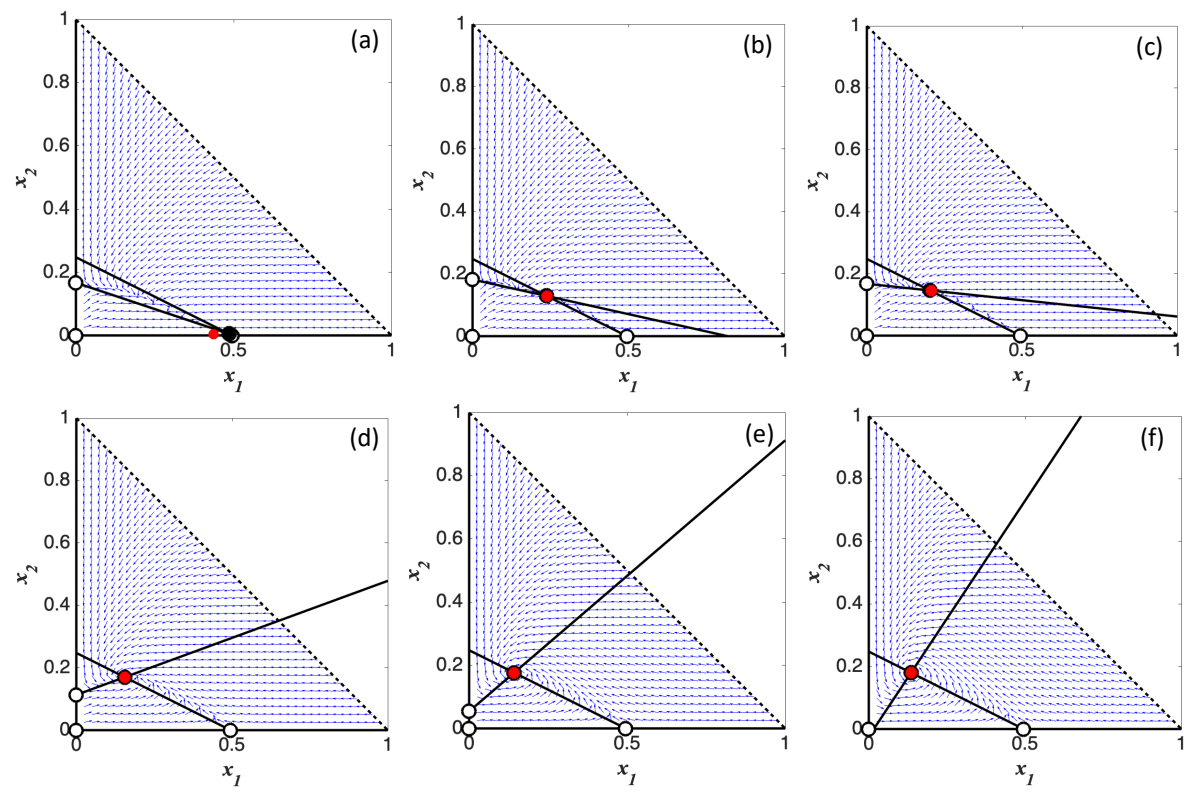

Fig. 1 Ecospace diagrams illustrating the ecosystem states $\left(\left\{x_{1}, x_{2}\right\}\right.$, red dots), zero isoclines (black lines), vector fields (blue arrows) and stable coexistence equilibrium outcomes (black dots) for the eco-evolutionary model described by equation (1) at: (a) commencement of integrations; (b) after 100 generations; (c) after 200 generations; (d) after 300 generations; (e) after 400 generations; (f) after 600 generations. See Table 1 for parameter values. Note that the $f_{2}=0$ isocline extending beyond $x_{1}=1$ in panel (c) denotes the initiation of net mixotrophy, and the positive slopes of the $f_{2}=0$ isocline in panels (d-f) indicates the dominance of heterotrophy. The translation of the $x_{2}$ intercept of the $f_{2}=0$ isocline down the $x_{2}$ axis in panels (d-f) indicates a reduction in autotrophy by $x_{2}$, until its location below the origin in panel (f) indicates the abandonment of autotrophy. Note that Figure 2 reveals that adaptation continues after 600 generations, but with little qualitative change to trophic interactions. Initial values for adapting parameters were $a_{21}=2.00$ and $\rho=0.9$.

by heterotrophy) (Cropp \& Norbury 2015a). This rapid response is completed by generation 300 (Figure 1(d)). The populations stabilise after 500 generations, each changing by just a few percent over the next 500 generations (Figure 2(a,d)). $x_{2}$ 's preference for autotrophy also remains relatively stable over the period of change, with $0.92<\rho<0.98$ for the first 200 generations, however, during this period of apparent stasis $x_{2}$ is adapting to a population that is more proficient at heterotrophy, with $a_{21}$ varying from about 2 to about -2 over this period.

After about 400 generations, the $x_{2}$ population begins to reduce its dependence on autotrophy, and increase its grazing upon $x_{1}$. During this transition in trophic strategy the equilibrium population sizes provide little clue of the adaptation and associated changes in $a_{21}$ in $x_{2}$. This transition continues in an approximately linear change until its net benefit from autotrophy becomes zero at about generation 1,000 . This leaves $x_{2}$ growing solely as a result of its grazing on $x_{1}$, that is, it has abandoned its mixotrophic life and has become a pure heterotroph.

An interesting attribute of the system evident in Figure 2 is the apparent disconnection between the dynamics of the evolutionary system and the dynamics of the ecological system. This is a result of the subtlety of the processes involved. 

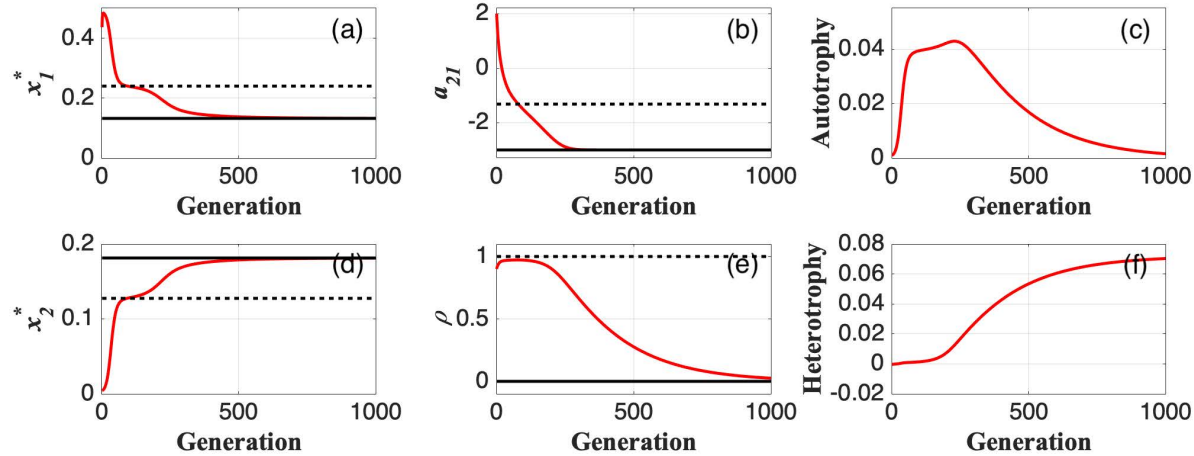

Fig. 2 Results obtained by integrating the system of equation (1) for 1,000 generations: (a) $x_{1}$ equilibrium value $x_{1}^{*}$; (b) evolution of $a_{21}$; (c) growth of $x_{2}$ by autotrophy; (d) $x_{2}$ equilibrium value $x_{2}^{*}$; (e) evolution of $\rho$; and (f) growth of $x_{2}$ by heterotrophy. The ecoevolutionary equilibrium point $E P_{0}$ is shown as solid black lines and $E P_{1}$ as dotted black lines in panels (a), (b), (d) and (e). The value of $a_{21}$ at which $\lambda_{4}$ changes sign is evident as the dashed line in (b) - $a_{21}$ attains this value at generation 80 . Note that $x_{2}$ rapidly evolves to be a better autotroph competitor, and then remains in apparent ecological stasis for about 200 generations while $a_{21}$ adapts. See Table 1 for parameter values. Note that Figure 1 reveals that the fundamental changes in trophic interactions are complete after about 600 generations, with the new arrangements effectively being optimised in the last 400 generations of the simulation. Initial values for adapting parameters were $a_{21}=2.00$ and $\rho=0.9$.

Figure 1 reveals that the adaptation initially involves rotation of the $f_{2}=0$ isocline, which almost pivots around the stable coexistence equilibrium point. The stable population sizes therefore provide no hint of the evolutionary processes that are under way. Although the population sizes barely change between generations 200 and $300, x_{2}$ is adapting its trophic strategy from a competing autotroph to a mixotroph, that both competes with and consumes $x_{1}$. Similarly, the stable population sizes between generations 500 and 1,000 belie the dramatic adaptation that is occurring within the $x_{2}$ population, in this case from a red mixotroph to a heterotroph.

The influence of the equilibrium points in the dynamics of the system is illuminated in Figure 2 by the dotted and solid black lines showing the locations of the equilibrium points. This reveals that the stable $\rho^{*}=0$ equilibrium point $E P_{0}$ does not immediately attract the dynamics of the system. It takes until about generation 200 at which stage $a_{21} \approx-2.7$ is approaching its bound $\left(-a_{12}=-3.00\right)$ and $\lambda_{4}=-0.15$ of $E P_{0}$ is approaching its greatest magnitude (-0.166) before the system begins to transition from $\rho=1$ to $\rho=0$. The ecological system has transitioned to $E P_{0}$ by around generation 500, at which time $a_{21}=-3.00$ has reached its bound but $\rho=0.25$ has yet to complete its transition to its equilibrium value (Figure 2). The evolutionary system does not complete the transition to $E P_{0}$ until about generation 1,000 .

Comparing generations 500 - 1,000 in Figures 1 and 2 reveals an interesting attribute of the evolutionary dynamics occurring in the system. The $x_{2}$ population reaches its "optimal" size $x_{2}^{*}=0.18$, an improvement from its pre-adaptation coexistence equilibrium of $x_{2}^{*}=0.004$, by about generation 500, having reduced the initial competitive advantage of $x_{1}^{*}=0.40$ to $x_{1}^{*}=0.13$. At this stage $x_{2}$ has adapted to be a "red mixotroph" that grows mostly by consuming $x_{1}$, as 
$r_{2} \rho N=0.18<0.20=a_{21}(1-\rho) x_{1}$. However, $x_{2}$ continues to adapt its trophic strategy, reducing its autotrophic growth and increasing its heterotrophic growth, without improving its equilibrium state, by rotating its $f_{2}=0$ isocline about the equilibrium point. This continued adaptation for no apparent benefit is a result of variance remaining in the distribution of feeding preference in the $x_{2}$ population - it continues to adapt until it reaches the equilibrium state associated with the stable eco-evolutionary point $E P_{0}$.

The fitness function (equation (4)) has the property that selection pressure still operates upon the populations when they are at ecological equilibrium. However, it is the natural trait bounds that determine whether adaptation occurs in a population. This is evident in Figure 2 where the populations are at ecological equilibrium for most generations. In the early period of population stability, from generations 60 to 600 , adaptation occurs because $a_{21}$ remains a substantial distance from its bound. However, $x_{2}$ does not utilise this new trophic strategy until the average trait is firmly established in the population, only commencing to grow substantially by consuming $x_{1}$ from about generation 300 , after it established the trait in the population.

Once the average trait value $a_{21}$ has closely approached its natural bound, it ceases to drive $x_{2}$ 's adaptation, and $x_{2}$ begins to exploit this new trophic strategy by changing its feeding preference $\rho$. This strategy slowly gains more prevalence, until from about generation 700 there is a rapid transition to heterotrophy taking over from autotrophy as the dominant feeding strategy, although the population sizes barely change in response to this dramatic change in food web structure. However, once this new state is reached, adaptation ceases as both average trait values lie near their natural bounds and the variances of the traits in the $x_{2}$ population are now near zero. This leaves the system primed to undertake further adaptation, perhaps in response to changes in the biotic or abiotic environment that move the natural bounds, or mutation within the populations that reintroduces variance.

When $a_{21}=-a_{12}$, and $x_{2}$ has "learnt" to very efficiently consume $x_{1}$ (i.e. $x_{2}$ increases its biomass by the entire amount it consumes of $x_{1}$ ), the stable heterotrophy equilibrium at $\rho=0$ dominates the behaviour of the system. Then as $a_{21}=-a_{12}$ the $\rho$ equation acts, with $N=1-x_{1}-x_{2}$ and $x_{1}, x_{2}=x_{1}^{E}, x_{2}^{E}$ (which involve $\rho$ ), so the evolution of $\rho$ from 1 to 0 (autotroph to heterotroph behaviour) also occurs on a very slow timescale. The key to the apparent disconnection between the decrease in $a_{21}$ that commences when $\rho=1$ is that crossing the hyperplane $r_{2} N=-a_{21} x_{1}$ changes the sign of $\lambda_{4}$, destabilising the $\rho=1$ state and stabilising the $\rho=0$ equilibrium. Crossing this hyperplane signifies that $x_{2}$ 's potential growth as a heterotroph exceeds its potential growth as an autotroph. While the transition from $\rho=1$ to $\rho=0$ occurs over many generations (about 750 in these simulations) the relatively rapid transition from $\rho \approx 0.8$ to $\rho \approx 0.2$ ) highlights the influence of the natural bounds on the trait values, and so on the evolutionary processes. Traits with average values distant from their bounds have the potential to evolve rapidly, where traits with average values near their bounds perforce evolve slowly. 
4 Conclusions

The numerical results show that a heuristic eco-evolutionary system (1) can simulate the evolution of pure heterotrophy in an autotroph population that has, as the green alga Chlamydomonas for example does, both autotrophic and heterotrophic metabolic pathways (i.e. that is a mixotroph (Flynn et al. 2013)). To the best of our knowledge, this is the first eco-evolutionary model to provide a mechanistic description of the emergence of heterotrophy. Importantly, the simplicity of the resource-based Lotka-Volterra model allows an analytic examination of the factors that control the dynamics evident in the numeric simulations.

Somewhat strikingly, the simulations show that the evolution of the autotroph to a heterotroph does not proceed monotonically, but has an intermediate stage where it improves its competitive functioning as an autotroph. Despite having the capability to function as a mixotroph, the initial adaptation of the mixotroph is to improve its autotrophic growth, and attempt to become a more effective autotroph competitor. Despite increasing its share of the total resources by a factor of 40 , it remains vulnerable, as its competitor sequesters around twice as much of the total resource as it does. It is only after pursuing this strategy for 500 generations or so, during which it optimises its mixotrophic potential, that the mixotroph adapts its feeding preference to transition from an autotroph through mixotrophy to a heterotroph.

A notable outcome of the numerical simulations is that the ecological state of the system does not necessarily reflect the evolutionary state, and vice versa. A dramatic change in the evolutionary status of the system may evidence itself as a minor change in the ecological status. For example, the dramatic change in the ecological state of the system, when $x_{1}^{*}$ reduces from holding $40 \%$ of the resources to $24 \%$, and $x_{2}^{*}$ increases from $0.4 \%$ to $15 \%$ over generations $1-100$, is accompanied by a similarly significant change in the ability of $x_{2}$ to behave as a mixotroph $a_{21} \rightarrow-1.5$. However, $x_{2}$ continues to function as an autotroph, increasing its autotrophic growth and maintaining its mixotrophic/heterotrophic growth near zero. It is only much later (generations 500-900) that the dramatic evolutionary change from autotrophy $\rho=1$ to heterotrophy $\rho=0$ in $x_{2}$ occurs, but this is accompanied by only a relatively subtle change in the ecological state, as $x_{1}^{*}$ reduces from $24 \%$ to $13 \%$ and $x_{2}^{*}$ increases from $15 \%$ to $18 \%$ of total resource. It is interesting to compare this observation with Bell's experimental result (Bell 2012) that algal systems appear adverse to abandoning autotrophy in favour of heterotrophy. However, perhaps extended duration experiments might produce the alternative conclusion that ultimately the algal system will effectively abandon autotrophy.

This modelling exercise emphasises the importance of natural bounds on trait distributions for eco-evolutionary modelling. The use of trait bounds is not new in eco-evolutionary modelling (for example, Abrams (1999), Cortez \& Patel (2017)) but the trait bounds used have often been trivial (for example, an efficiency bounded between 0 and 1), intuitive (a trait mean bounded away from 0 ), or sometimes arbitrary. As far as we are aware, this work is the first to use trait bounds that arise from fundamental properties of the ecological model. These bounds effectively eliminate degenerate behaviours from the model solutions - the model cannot represent immortal populations, neither does it consider unviable populations that cannot grow under any scenario. The bounds also ensure that physically 
unrealistic processes, such as a predator gaining more mass from predation than its prey loses, cannot occur.

The bounds imply distributions for which the variance of the trait is a function of the distance of the trait average from the natural bound. It is the natural bounds, that in many cases maintain specific relationships between average values of different traits, that constrain the adaptation of the population to ecologically valid outcomes. The natural trait bounds have been implemented mathematically in the model; consequently the average trait values are capable of approaching the bounds infinitesimally closely in the computer simulations. Physiological constraints in real organisms are likely to operate to maintain actual average trait values further away from their natural bounds than would occur in our mathematical idealisation. Alternatively, a population may have the physiological capacity to exceed its theoretical bounds, in which case non-ecological outcomes such as self-extinction might eventuate.

The idealised model of eco-evolutionary processes here shows the emergence of heterotrophy for a population (allegorically a green alga) that has metabolic pathways that allow it to utilise both inorganic and organic resources. The emergence of pure heterotrophy is a necessary and key step in building up trophic levels in food webs and ecosystems. The model simulations suggest that the evolution of a new trophic level does not necessarily occur monotonically or uniformly, and that the signatures of such transitions in field observations may be subtle.

\section{Conflicts of Interest}

The authors declare no conflicts of interest.

\section{Acknowledgements}

The authors thank two very thorough and constructive anonymous reviewers for their contributions to publishing this manuscript.

\section{Appendix}

\subsection{Appendix One: Equilibrium Points}

The full eco-evolutionary model (1) has eco-evolutionary coexistence equilibrium points $\left\{x_{1}^{*}, x_{2}^{*}, a_{21}^{*}, \rho^{*}\right\}$, that are potentially stable and are the focus of our investigation. One coexistence equilibrium point $E P_{1}$ is:

$$
\begin{aligned}
x_{1}^{*} & =\frac{r_{1} a_{22}-r_{2} a_{12}-m_{1}\left(r_{2}+a_{22}\right)+m_{2}\left(r_{1}+a_{12}\right)}{r_{1} a_{22}-r_{2} a_{12}+a_{11}\left(r_{2}+a_{22}\right)}, \\
x_{2}^{*} & =\frac{r_{1}\left(1-x_{1}^{*}\right)-m_{1}-a_{11} x_{1}^{*}}{r_{1}+a_{12}}, \\
a_{21}^{*} & >-a_{12}, \\
\rho^{*} & =1 .
\end{aligned}
$$


For the parameter values used in the simulations (Table 1) this point lies inside the feasible ecospace $E \equiv\left\{0<x_{1}<1,0<x_{2}<1 ; 0<x_{1}+x_{2}<1\right\}$, and is relevant to the early eco-evolutionary dynamics of the system. Note that when $\rho^{*}=1$ the value of $a_{21}^{*}$ is not fixed as it does not appear in the model equations (1) or (13). Substituting $\rho^{*}=1$ into the Jacobian matrix (equation (9)) of the model (equation (1)) and solving for the eigenvalues gives:

$$
\begin{aligned}
& 2 \lambda_{1,2}=-\left(r_{1}+a_{11}\right) x_{1}^{*}-\left(r_{2}+a_{22}\right) x_{2}^{*} \pm \sqrt{\begin{array}{c}
\left\{\left(r_{1}+a_{11}\right) x_{1}^{*}-\left(r_{2}+a_{22}\right) x_{2}^{*}\right\}^{2} \\
+4 r_{2}\left(r_{1}+a_{12}\right) x_{1}^{*} x_{2}^{*}
\end{array}}, \\
& \lambda_{3}=0 \text {, } \\
& \lambda_{4}=-\frac{h_{2}^{2}}{\beta_{2}^{2}}\left(r_{2} N^{*}+a_{21}^{*} x_{1}^{*}\right) \text {. }
\end{aligned}
$$

As the value of $a_{21}^{*}>-a_{12}$ is not fixed for $E P_{1}$, in the four dimensional eco-evo phase space this equilibrium point in $E$ becomes a half-line of equilibrium points. Further, $\lambda_{4}$ can change sign and its stability along the $\rho$ manifold can change, and is negative when $a_{21}^{*}$ is sufficiently positive, that is when $a_{21}^{*}>-r_{2} N^{*} / x_{1}^{*}$.

The other coexistence equilibrium point $E P_{0}$ is:

$$
\begin{aligned}
x_{1}^{*} & =\frac{r_{1}\left(m_{2}+a_{22}\right)-m_{1} a_{22}+m_{2} a_{12}}{a_{11} a_{22}-a_{12} a_{21}^{*}-r_{1}\left(a_{21}^{*}-a_{22}\right)}, \\
x_{2}^{*} & =\frac{r_{1}\left(1-x_{1}^{*}\right)-m_{1}-a_{11} x_{1}^{*}}{r_{1}+a_{12}}, \\
a_{21}^{*} & =-a_{12}, \\
\rho^{*} & =0 .
\end{aligned}
$$

The eigenvalues of this point are:

$$
\begin{aligned}
2 \lambda_{1,2} & =-\left(r_{1}+a_{11}\right) x_{1}^{*}-a_{22} x_{2}^{*} \pm \sqrt{\begin{array}{c}
\left\{\left(r_{1}+a_{11}\right) x_{1}^{*}-a_{22} x_{2}^{*}\right\}^{2} \\
+4 r_{2}\left(r_{1}+a_{12}\right) x_{1}^{*} x_{2}^{*}
\end{array}}, \\
\lambda_{3} & =-\frac{h_{1}^{2}}{\beta_{1}} x_{1}^{*}, \\
\lambda_{4} & =\frac{h_{2}^{2}}{\beta_{2}^{2}}\left(r_{2} N^{*}-a_{12} x_{1}^{*}\right) .
\end{aligned}
$$

The eigenvalues $\lambda_{1,2}$ on the $\left\{x_{1}, x_{2}\right\}$ manifold remain the same, and are negative for all values used in the numerical simulation. The eigenvalue along the $a_{21}$ manifold $\lambda_{3}$ is always negative for positive values of $x_{1}$, and the eigenvalue along the $\rho$ manifold $\lambda_{4}$ is also negative as $r_{2} N^{*}<a_{12} x_{1}^{*}$. This point is then stable. Note that the fourth eigenvalues $\lambda_{4}$ of $E P_{1}$ and $E P_{0}$ have opposite signs. 
Table 1 Parameter values used in equation (1) to draw Figures 1 and 2.

\begin{tabular}{lll}
\hline Parameter & Process & Value \\
\hline$r_{1}$ & Maximum $x_{1}$ autotroph growth rate & 1.00 \\
$m_{1}$ & Density-independent mortality rate of $x_{1}$ & 0.01 \\
$r_{2}$ & Maximum $x_{2}$ autotroph growth rate & 0.50 \\
$m_{2}$ & Density-independent mortality rate of $x_{2}$ & 0.125 \\
$a_{11}$ & Density-dependent mortality rate of $x_{1}$ & 1.00 \\
$a_{12}$ & Effect of $x_{2}$ upon $x_{1}$ & 3.00 \\
$a_{21}$ & Effect of $x_{1}$ upon $x_{2}$ & $-3.00 \rightarrow 2$ \\
$a_{22}$ & Density-dependent mortality rate of $x_{2}$ & 1.50 \\
$h_{1}$ & Realised additive heritability of $a_{21}$ & 0.50 \\
$h_{2}$ & Realised additive heritability of $\rho$ & 0.075 \\
$\beta_{1}$ & Shape parameter of $a_{21} \gamma$-distribution & 0.25 \\
$\beta_{2}$ & Shape parameter of $\rho \gamma$-distribution & 0.25 \\
$\rho$ & Feeding preference of $x_{2}$ & $0 \rightarrow 1$ \\
\hline
\end{tabular}

\subsection{Appendix Two: Parameter Values}

Although this work is conceptually based on the results of Bell (2012), the ecological model is heuristic, with population interactions represented in their simplest form, so we have not attempted to reproduce the timescale of evolution noted by Bell. The parameter values have been chosen to produce interesting, representative dynamics in a reasonable computational time. We do not report a parameter sensitivity analysis here, but note that the evolutionary time scales are sensitive to both the evolutionary and ecological parameters. In particular, we note that the $x_{2}$ density-independent mortality parameter $m_{2}$ has a significant effect on the evolutionary timescale, supporting the view that adaptation is driven by the rate at which less-fit individuals are removed from the population.

\section{References}

Abrams, P. (1991), 'The effects of interacting species on predator-prey coevolution', Theor Pop Biol 39, 241-262.

Abrams, P. (1999), 'The adaptive dynamics of consumer choice', Am Nat 153, 8397.

Abrams, P. \& Matsuda, H. (2004), 'Consequences of behavioral dynamics for the population dynamics of predator-prey systems with switching', Popul Ecol 46, 13-25.

Abrams, P., Matsuda, H. \& Harada, Y. (1993), 'Evolutionarily unstable fitness maxima and stable fitness minima of continuous traits', Evol Ecol 7, 465-487.

Bell, G. (2012), 'Experimental evolution of heterotrophy in a green alga', Evolution 67(2), 468-476.

Bengtson, S. (2002), 'Origins and early evolution of predation', Paleontological Society Papers 8, 289-318.

Chesson, P. (2000), 'Mechanisms of maintenance of species diversity', Annu. Rev. Ecol. Syst 31, 343-366.

Cortez, M. (2016), 'How the magnitude of prey genetic variation alters predatorprey eco-evolutionary dynamics', Am Nat 188, 329-341. 
Cortez, M. \& Ellner, S. (2010), 'Understanding rapid evolution in predatorprey interactions using the theory of fast-slow dynamical systems', Am Nat 176(5), E109-E127.

Cortez, M. \& Patel, S. (2017), 'The effects of predator-evolution and genetic variation on predator-prey population-level dynamics', B Math Biol 79, 1510-1538.

Coutinho, R., Klauschiies, T. \& Gaedke, U. (2016), 'Bimodal trait distributions with large variances question the reliability of trait-based aggregate models', Theor Ecol 9, 389-408.

Cropp, R. \& Norbury, J. (2015a), 'Mixotrophy: the missing link in consumerresource-based ecologies', Theor Ecol 8, 245-260.

Cropp, R. \& Norbury, J. (2015b), 'Population interactions in ecology: A rule-based approach to modeling ecosystems in a mass-conserving framework', SIAM Rev $\mathbf{5 7}(3), 437-465$.

Cropp, R. \& Norbury, J. (2019), 'An eco-evolutionary system with naturally bounded traits', Theor Ecol pp. doi:10.1007/s12080-019-0407-6.

De Meester, L., Brans, K., Govaert, L., Souffreau, C., Mukherjee, S., Vanvelk, H., Korzeniowski, K., Kilsdonk, L., Decaestecker, E., Stoks, R. \& Urban, M. (2019), 'Analyzing eco-evolutionary dynamics - the challenging complexity of the real world', Functional Ecology pp. doi:10.1111/1365-2435.13261.

Flynn, K., Stoecker, D., Mitra, A., Raven, J., Glibert, P., Hansen, P., Graneli, E. \& Burkholder, J. (2013), 'Misuse of the phytoplankton - zooplankton dichotomy: the need to assign organisms as mixotrophs within plankton functional types', J Plankton Res 35, 3-11.

Fussman, G., Ellner, S. \& Hairston, N. J. (2002), 'Evolution as a critical component of plankton dynamics', P R Soc London B Bio 270, 1015-1022.

Fussman, G., Loreau, M. \& Abrams, P. (2007), 'Eco-evolutionary dynamics of communities and ecosystems', Functional Ecology 21(3), 465-477.

Gaedke, U. \& Klauschies, T. (2017), 'Analysing the shape of observed trait distributions enables a data-based moment closure of aggregate models', LEO Methods 15, 979-994.

Govaert, L., Fronhofer, E., Lion, S., Eizaguirre, C., Bonte, D., Egas, M., Hendry, A., De Brito Martins, A., Melian, C., Raeymaekers, J., Ratikainen, I., Saether, B., Schweitzer, J. \& Matthews, B. (2018), 'Eco-evolutionary feedbackstheoretical models and perspectives', Functional Ecology pp. doi:10.1111/13652435.13241.

Klauschies, T., Coutinho, R. \& Gaedke, U. (2018), 'A beta distribution-based moment closure enhances the reliability of trait-based aggregate models for natural populations and communities', Ecological Modelling 381, 46-77.

Lande, R. (1976), 'Natural selection and random genetic drift in phenotype evolution', Evolution 30, 314-334.

Mitra, A., Flynn, K., Burkholder, J., Berge, T., Calbet, A., Raven, J., Graneli, E., Glibert, P., Hansen, P., Stoecker, D., Thingstad, F., Tillmann, U., Vage, S., Wilken, S. \& Zubkov, M. (2014), 'The role of mixotrophic protists in the biological carbon pump', Biogeosciences 11, 995-1005.

Osmond, M., Otto, S. \& Klausmeier, C. (2017), 'When predators help prey adapt and persist in a changing environment', Am Nat. 190, 83-98.

Pastor, J. (2017), 'Ecosystem ecology and evolutionary biology, a new frontier for experiments and models', Ecosystems 20, 245-252. 
Pelletier, F., Garant, D. \& Hendry, A. (2009), 'Eco-evolutionary dynamics', Phil Trans Roy Soc B 364, 1483-1489.

Post, D. \& Palkovacs, E. (2009), 'Eco-evolutionary feedbacks in community and ecosystem ecology: interactions between the ecological theatre and the evolutionary play', Phil Trans Roy Soc B 364, 1629-1640.

Schoener, T. (2011), 'The newest synthesis: Understanding the interplay of evolutionary and ecological dynamics', Science 331, 426-429.

Selosse, M., Charpin, M. \& Not, F. (2017), 'Mixotrophy everywhere on land and in water: the grand ecart hypothesis', Ecology Letters 20, 246-263.

Vasseur, D., Amarasekare, P., Rudolf, V. \& Levine, J. (2011), 'Eco-evolutionary dynamics enable coexistence via neighbor-dependent selection', The American Naturalist 178(5), E96-E109.

Vitousek, P. \& Matson, P. (2012), Nutrient cycling and biogeochemistry, in S. Levin, ed., 'The Princeton Guide to Ecology', Vol. 1, Princeton University Press, Princeton New jersey, pp. 330-339. 